\title{
INVESTIGATION OF UTRA FDD DATA AND Control Channels in the Presence of Noise AND STANDARD DEFINED CHANNEL MODELS
}

\author{
Ibrahim Aref \\ Computer Engineering Department, University of Tripoli, Tripoli, Libya \\ iarefetripoliuniv.edu.ly
}

\begin{abstract}
In this paper, the main aim is to design and simulate UTRA FDD control channel in the presence of noise and wireless channel by using FDD library/Matlab box set that can be used to design and implement some systems. Moreover, a test and verification of the library is achieved with different channel models such as Additive White Gaussian Noise (AWGN), fading and moving channel models. FDD library are employed to design whole transmitter and receiver. Then we had tested AWGN channel and some other channel models. Also we illustrated what are control channels DCCH and the other one as understanding the whole system. Moreover, the standards have been covered as well as implemented the whole transmit and receive chain plus the generation of DPCH, DPCCH channel. we had tested the performance against the AWGN noise. Then we have studied different channel models that are defined in the standard, used the few of them like the fading channel and moving channel. We have tried to compare the performance in terms of Monte Carlo simulation by producing the BER curves. We have also change some channel parameters like phase, number of multipaths and we have tried to see the performance of the model in the presence of actual channel model.
\end{abstract}

\section{KEYWORDS}

Modelling, Simulation, UTRA, FDD, Channel

\section{INTRODUCTION}

The cellular wireless communications industry has been one of the fastest growing technologies in the past decade. Up to now, there are over four billion wireless subscribers worldwide. From first generation $(1 \mathrm{G})$ till third generation $(3 \mathrm{G})$, the communication systems performance is improved with the appearance of new technologies. At the present time, wireless communication becomes a very important technology and has appeared in the market since it provides a set of good features to the network users such as network mobility, scalability and connectivity. Wireless networks are completely different from wired networks. In wired networks the signal propagates through fixed time invariant medium such as cables, fibre optics etc. In case of wireless communication the signal propagates through time varying channel i.e. the characteristic of the propagation medium changes due to user mobility and terrain conditions. In the cellular communication system, a large number of transmitters but with low power value are employed to form cells. The cell represents the small geographic service region of the cellular system. The basic and main requirement of the cellular system is the high capacity that can be performed by confining the coverage of the all stations (base stations) to the cell size [1]. This is different from traditional radio transmitter which would cover the entire city and whole city was one cell. So in 
order to achieve higher capacity that is to support a large number of user, the city must be divided into a smaller cells and each cell must have a transmitter tower called base station (BS). The customer can move around the cells regions (to go from one cell location to another), and then the call will not be interrupted, because it is enabled by a switching techniques called hand-off $[1,2]$. In this work, the focus will be on cellular mobile communications that introduced around 1980 based on the radio system technology and known as cell phones. At that time, the first generation (1G) of wireless communications systems are presented and supported voice communications with limited roaming [2]. A set of wireless standard introduced such as Advanced Mobile Phone System (AMPS) [3]. AMPS is a $1 \mathrm{G}$ analogue mobile phone system standard that is expanded and improved by Bell Labs and introduced in America in 1983, with differing such as Total Access Communication System (TACS) available in Europe and Japanese Total Access Communication (JTAC) available in Japan [4]. Most of these standards are based on frequency division multiplexing (FDM) scheme which allocate each mobile user with different carrier frequency. In FDM, data from different transmitters are sent at the same time but over multiple frequencies. Each one of the sub-carriers frequencies is modulated by different data stream. In order to avoid adjacent channel interference, there is a guard band between sub-carriers [5]. The main aim of this work is to understand and simulate UTRA FDD control channel in the presence of noise and wireless channel.

This paper is organised in four sections. In section one, an introduction and an overview of the $3 \mathrm{G}$ technologies. Section two gives an idea about UTRA FDD Physical Layer. The simulation models of FDD and results are illustrated in section three. Finally, conclusions are presented in section four.

\section{UTRA FDD PHYSICAL LAYER}

3GPP introduced the first release of universal terrestrial radio access (UTRA) FDD air interface in 1999. The UTRA radio access is based on wideband code division multiple access (WCDMA) and hybrid time-division (TDCDMA). This release is specified with two antenna open-loop and closed-loop transmits diversity modes. In this section, we will cover the UTRA physical layer [6].

\subsection{UTRA Physical Layer}

UTRA physical layer offer two ways of the implementations. The first one can be achieved by using FDD and the other one by using TDD. Each approach satisfies different needs and requirements. Briefly in this section, we will give an overview for each one.

\subsubsection{FDD}

FDD specifies two frequency bands that can be used for uplink and downlink. So its main advantage is to be able to send and receive at the same time. Moreover, the cell size is not depended by propagation delays because FDD didn't have a guard periods and time slots. Also for this reason the timing synchronisation between base station and mobiles users less critical than TDD. In other hand, FDD is more complicated that TDD because FDD radio units require duplexers that can be employed to separate the incoming and outgoing signals at the antenna and then FDD can send and receive at the same time [7]. Duplexers are created of filters, so this is the reason to increase complexity and hardware components cost. Moreover, there is a disadvantage which is the bandwidth couldn't be allocated in efficient way, because there is some service types required more throughput on the downlink such as Internet access and other service required more throughput on the uplink. Of course, it may possible to use just the required data rate by adjusting the spreading factor, but it is still impossible to trade uplink BW for downlink BW [8]. 


\subsubsection{TDD}

TDD is the Time Division Duplex mode. Here downlink and uplink employ the same frequency band but they used different time slots. The time that specified for Dl or UL is divided into frames and also each frame is divided into a group short durations known as time slot. All the time slots could be allocated to DL or to the UL. So for the link that needs more throughputs, TDD allocates more time slots for this link. Here the big advantage is the duplexer is not required, which means that TDD becomes less complexity that TDD. But in the other side, TDD needs more synchronization between its terminals that FDD that is based on time division concept (the base station doesn't have the capabilities to send data at the same time as the mobile) [8].

\subsection{UTRA Framework}

In wideband code division multiple access, narrowband user data is distributed over a wide bandwidth. This can be achieved by modulating user data sequence through very slow rate with a channelization code in high rate. The spreading codes range starting from 4 up to 512 chips. In case of uplink, the maximum number of chips (code length) is 256 chips. The rate of transferring chips (rate of the chip) is 3.84 Mcps (Mega chips per second). This can be covered a bandwidth of $5 \mathrm{MHz}$. Moreover, both Variable spreading factors and multi-code transmission can be employed to support different data rates [6].

Length 38400 chips from scrambling codes are used on top of channelization codes. In the downlink, the channelization code is employed to divide various intra-cell users. This separation is based on various scrambling codes. On the other hand (uplink), the scrambling codes separate different users. Also, channelization codes divide various physical data and control channels of a user. This variation in the case of downlink and uplink is necessary:

- The orthogonal channelization codes number is restricted, os it makes a strict upper bound to the achievable downlink capacity.

- In case of uplink, the restriction is not precise, because for example, a user can be used all channelization codes and the scrambling codes set includes some million codes.

The data rates that can be supported by UTRA FDD basic mode is closed to $2.3 \mathrm{Mbps}$ which included both cases (uplink and downlink). In the downlink, the sending data with maximum rate requires the employ of three parallel channelization codes and spreading factor equal 4 , that means, it assigns up to $75 \%$ of code resources to just a one user. In uplink case, sending data with the peak rate is seen as a distortion or noise source in the communication network, but present code of other intra-cell users are not changed.

\subsubsection{UTRA FDD Downlink}

The frame structure of the downlink channel is contained of 15 time slots; each with a length of $10 \mathrm{~ms}$. Figure 1 shows a downlink frame structure of the physical channel. The radio frame provides the following channels [6]:

1. Dedicated physical channel (DPCH): It is divided into dedicated physical data channel (DPDCH) and dedicated physical control channel (DPCCH).

2. control channel : It contains the following :

- Transmit power control (TPC) bits that can be used for fast power control.

- Transport format combination indicator (TFCI) bits that used to tell the receiver about transport channels states, which channel is active or not. 
- Pilot bits enabling the channel and signal to interference and noise ration (SINR) estimation from DPCH.

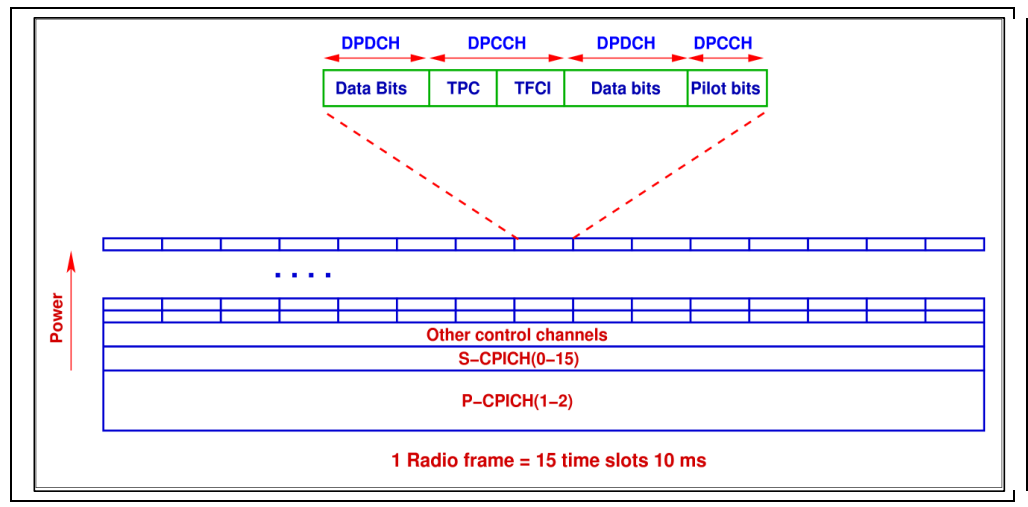

Figure 1. Downlink frame structure for dedicated physical channel

The primary common pilot channel (P-CPICH) and the secondary common pilot channel (S$\mathrm{CPICH}$ ) are shown in Figure 1. The numbers in parentheses state that one sector in downlink contain 1-2 parallel P-CPICHs and 0-15 parallel S-CPICHs.

Based on the specification of the UTRA FDD, there is just two P-CPICHs are ready to use at maximum. Also multiple S-CPICHs can be presented, for example as beamforming purposes. This presents a serious order compatibility problem in case of designing MIMO algorithms. If the power in P-CPICH is constant (not subject to change) and it is distributed between two or more transmit antennas, so the channel estimation in legacy mobile terminals is decayed. If the power of P-CPICH is increased, interference to network increases as well $[8,9]$.

\subsubsection{UTRA FDD Uplink}

The uplink frame and time slot of the DPDCH and DPCCH are created as illustrated in the Figure 2. In the case of the uplink, control information and user data are constructed as I/Q code multiplexed and some of DPDCHs could be integrated to a single DPCCH. Besides pilot bits, a feedback information bits (FBI) are included with the TFCI and TPC uplink DPCCH slot. These bits can be used to carry the state of the partial channel information. This can be done in case of UTRA two-antenna closed-loop transmits diversity modes. In the uplink, only the user-specific dedicated pilot channels are available in uplink. The users in the uplink are not synchronised. Also the multi-user interference is not avoided, because user's channelization codes are nonorthogonality[6,8].

\subsection{UTRA FDD Physical Layer}

\subsubsection{Channels}

The main function of the FDD Physical Layer is to transfer data and information to the other protocol layers. This can be achieved by using channel. Here, there are two channels, the first one is Transport Channels and the other one is Physical Channels.

1. Transport Channels are responsible to represent the characteristics of the data that will be transmitted through the radio interface. The characteristics such as: 
- Format: Bit rate, encoding (convolution, turbo codes), interleaving.

- Framing / Multiplexing: This means how we can multiplex data if it created from different sources (more than one).

- General Features: Power control features, collision possibility, Technique the used to defined mobile station, Beam forming chance, Data rate changing, Broadcast region (whole cell or just specific sector), Uplink or Downlink.

3. Physical Channel is responsible to describe the spectrum and code that employed by the radio channel. Also in uplink case, it can state the relative phase, if it is In-Phase or Quadrature.

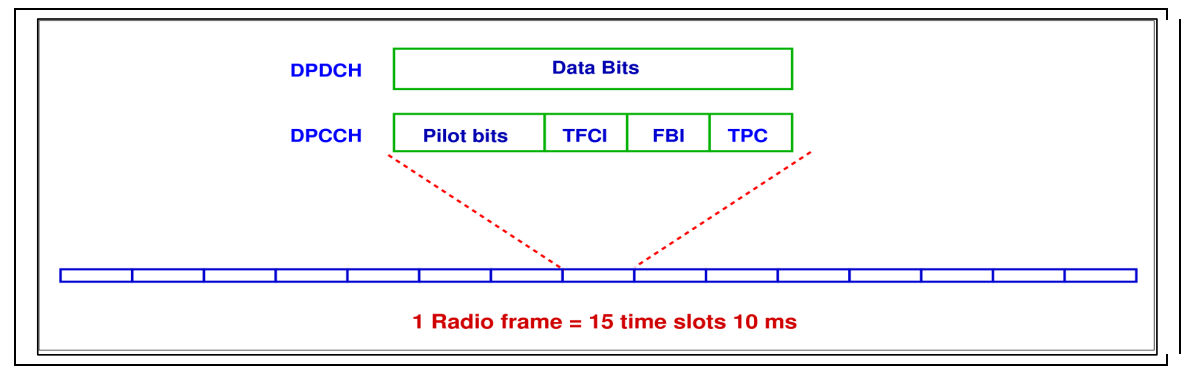

Figure 2. Uplink frame structure for DPDCH/DPCCH

\subsubsection{Transport Channels}

Here we will state the channel types. Transport channels have two types. The first one is known as Dedicated Transport Channels and the other one is known as Common Transport Channels.

\subsubsection{Physical Channels}

The physical channels are the representation of the transport channel, because we need to transmit data through the air by using channel. In the uplink path, the physical channel is represented by the following parameters [6]:

- Frequency, which means the carrier frequency.

- Channelisation code.

- Relative phase, if it is (0) means in-Phase or if it is (PI/2) which means Quadrature component.

\subsubsection{Channelisation Codes}

In CDMA system, there are codes that can be employed in the channels separation process. These channels may use a fixed frequency. It is important to select the suitable channelisation codes that are orthogonal. So the system can support a different data rates and SF. UTRA employs Orthogonal Variable Spreading Factor (OVSF) codes. There is a various values of the channelisation codes. It is depended on the number of the physical channel and data rate. As indicated in RTT manual, the voice channel number per carrier equal 250, which means, orthogonal codes (256) take away some control channels.

\subsubsection{Scrambling Codes}

Scrambling operation is defined as spread chips multiple PN sequence. Using this operation, the 
signal spectral features will be improved by virtue of the PN. In the UTRA system, Kasami and Gold sequences are used. Both of them are two families of PN sequences.

\subsubsection{Modulation and Spreading}

\subsubsection{Uplink}

In this section, we will start to describe the modulation and spreading process of uplink UTRA FDD as shown in the Figure 3. As shown in this figure, $\boldsymbol{C c}$ and $\boldsymbol{C d}$ are used to describe channelisations codes. Each one is set to the different branch. The codes $\boldsymbol{C} \boldsymbol{c}$ and $\boldsymbol{C d}$ are defined as OVSF codes and they are working at the chip rate. They can be employed just in one mobile station. Cscramb is another parameter and it is known as a complex scrambling code. It is operating at the chip rate. In the uplink path, each mobile should be employed a different scrambling code. The same diagram is also can be employed to send the PRACH. The data packet and the control packet are separated and both occur of the DPDCH and DPCCH.

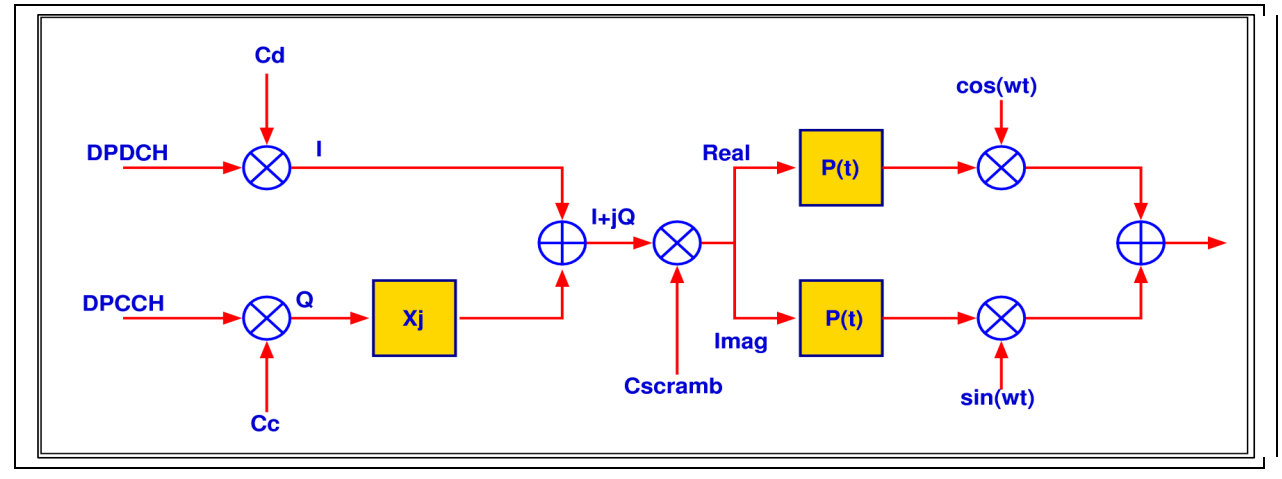

Figure 3. UTRA FDD uplink modulation and spreading

\subsubsection{Downlink}

In the downlink case, the modulation and spreading techniques are a little bit different when compared to the case of the uplink path. The modulation and spreading for the downlink DPCH is described in the Figure 4. The serial bits are changed into a parallel and set to the In-Phase and Quadrature branch. After that, both are distributed at the chip rate by using the same channelisation code and scrambling code.

\section{Simulation AND RESUltS}

In this section, a simulation model of FDD downlink channel is developed using DPCH (Dedicated Physical Channel) from two transport channels (DTCH at $64 \mathrm{kbps}$ and DCCH at 2.5 kbps) is developed using FDD library. The simulated downlink is tested using Gaussian noise source and standard ITU channel models. The simulation model is developed using Maths works Matlab/Simulink environment using UTRA FDD library developed by Steepest Ascent. A Matlab Simulink program is used to build complete model of FDD model from transmitter to receiver end and to investigate FDD system performance. 


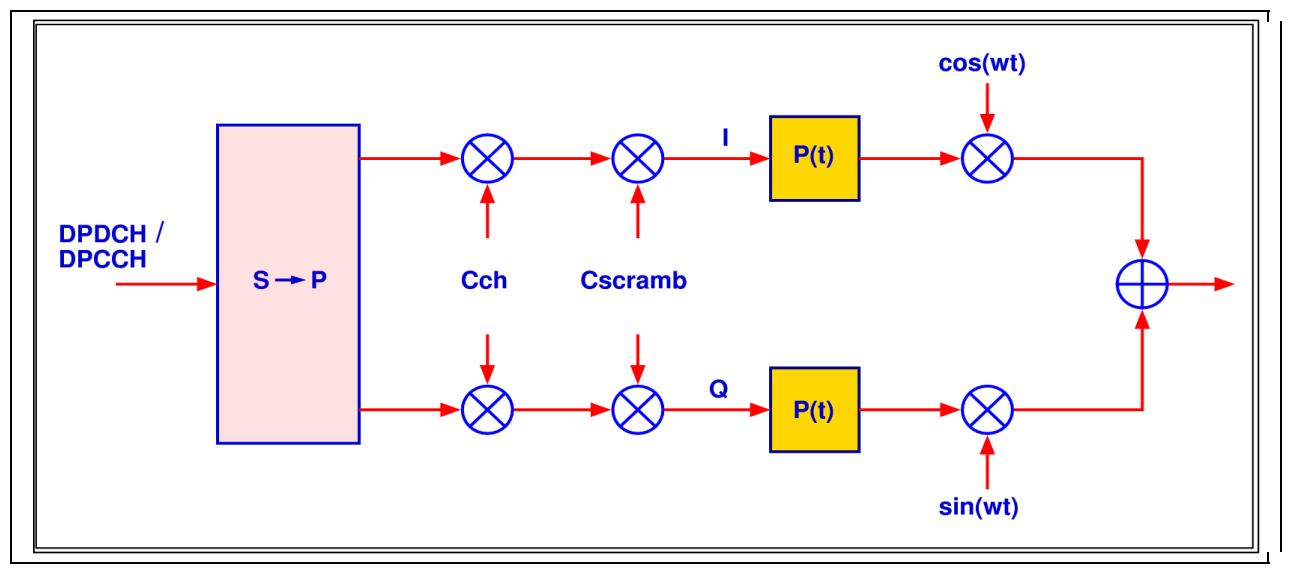

Figure 4. UTRA FDD downlink modulation and spreading

\subsection{Simulation Procedure}

In the first step of this simulation, the transmitter is used to create a digitally modulated signal from a sequence of pseudo-random bits. After that, we have to do some measurements over that signal $\times(n)$. The signal-to-noise-ratio $(\mathrm{SNR}), \mathbf{E}_{\mathbf{b}} / \mathbf{N}_{\mathbf{o}}$, is usually expressed in decibels; the logarithmic values are converted to linear scale in the simulation model using the following equation.

$$
\frac{E_{b}}{N_{0}}=10^{\frac{m}{10}}
$$

\subsubsection{Determine $\mathbf{E}_{b}$}

Energy-per-bit can be defined as the total energy of the signal, divided by the number of bits comprised in the signal. Also energy-per-bit can be represented as the average signal power multiplied by the duration of one bit. Either way, the expression for $\mathbf{E}_{\mathbf{b}}$ is:

$$
E_{b}=\frac{1}{N \cdot f_{b i t}} \sum x^{2}(n)
$$

Where $\mathbf{N}$ is the total number of samples in the signal, and $\mathbf{f}_{\mathbf{b i t}}$ is the bit rate in bits- per-second.

\subsubsection{Calculate $\mathbf{N}_{\mathrm{o}}$}

In this stage, No can be evaluated by using the SNR and energy-per-bit as well as we can find the one-sided power spectral density of the noise by using the same parameters. We need to divide $\mathbf{E}_{\mathbf{b}}$ by the SNR, providing we have converted the SNR from decibels to a ratio. The units of the power spectral density noise can be expressed as Watts per Hertz.

The bit error rate is calculated using monticarlo simulation where a fixed set of bits are transmitted. When the simulation of the bit-error-rate is executed; the numbers of bits that transmitted and received have to be fixed. The errors in the received bits are evaluated, and then we can find the bit-error-rate by dividing the number of bits errors over the total bits of the transmitted signal. The simulation process of the bit-error-rate can be a long task. It is better to 
execute an individual simulation at each SNR of interest. Moreover we need to check that our results are statistically significant.

\subsection{Statistical Validity}

If the value of the bit-error-rate is large, which means the error occurs within a large number of bits. The worst-case bit-error rate is 50 percent, at which point, the modem is essentially useless. The bit-error-rates in many communications systems should be lower than this value.

Most of the time needs to plot a curve of the bit-error-rate as a function of the SNR, also we have to add sufficient number of points in order to cover a wide range of bit-error-rates. At high SNRs, this can become difficult, since the bit-error-rate becomes very low. For example, if the value of the bit-error-rate is equal 10-6 that means only one bit out of every 106 bits will be in error.

If our test signal only contains 1000 bits, we will most likely not see an error at this bit-error-rate. In order to be statistically significant, each simulation we run must generate some number of errors. If the output of the simulation process produces no errors, it does not mean the bit-errorrate is equal to zero; but that means we did not transmit a sufficient number of bits in our signal. As a rule of thumb, we require more than or equal 100 errors in each simulation process, in order to get confidence that our bit-error-rate is statistically valid. At high SNRs, in this case we need to check the signal that containing millions, or even billions of bits.

\subsection{Plotting}

If we execute enough simulations in order to obtain valid results at all SNRs, then we can plot the results. We start by making vectors for $\mathrm{X}$-axis and $\mathrm{Y}$-axis. The $\mathrm{X}$-axis vector will include $\mathrm{SNR}$ values, while the other one which is $\mathrm{Y}$-axis vector will include bit-error-rates. The $\mathrm{Y}$ - axis can be plotted on a logarithmic scale, whereas the $\mathrm{X}$-axis will be plotted on a linear scale. Supposing our SNR values are in vector 'xx', and our corresponding bit-error-rate values are in vector 'yy', so we can use Matlab to plot by using the following statement:

semilog ( $\left.x \mathbf{x}, y \mathbf{y}, \mathrm{O}^{\prime}\right)$

\subsection{Simulation setup model for BPSK using AWGN channel}

Simulation model for binary phase shift keying (BPSK) is shown in the Figure 6. In this simulation a BPSK signal is generated and transmitted through a AWGN channel. The AWGN Channel block adds white Gaussian noise to a real or complex input signal. If the input signal is real, then the block adds real Gaussian noise and produces a real output signal. In the case of a complex input signal, this block will add complex Gaussian noise and then generates a complex output signal (Figure 5).

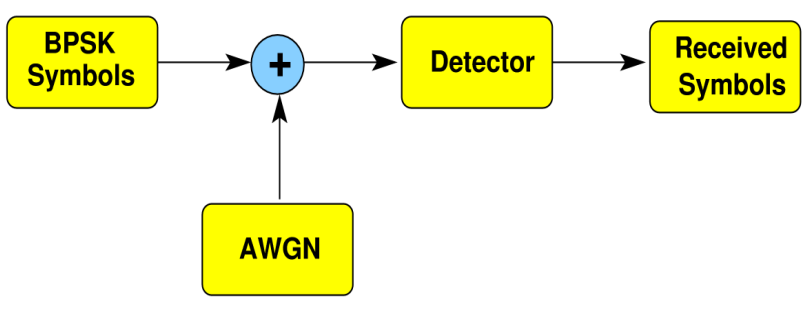

Figure 5. Block diagram of BPSK module 


\subsubsection{Steps}

1. The model generates BPSK symbols which are passed through AWGN channel.

2. The received signal is detected using a simple comparator.

3. The BER is calculated based on transmitted and received bits.

4. Keep the M file and the BPSK model in the same directory before execution

5. Run the model first to generate the theoretical BER curves

6. Draw the BER curves with AWGN.

\subsubsection{Result}

The BER obtained using Matlab/Simulink scripts show good agreement with the theory as shown in Figure 7.

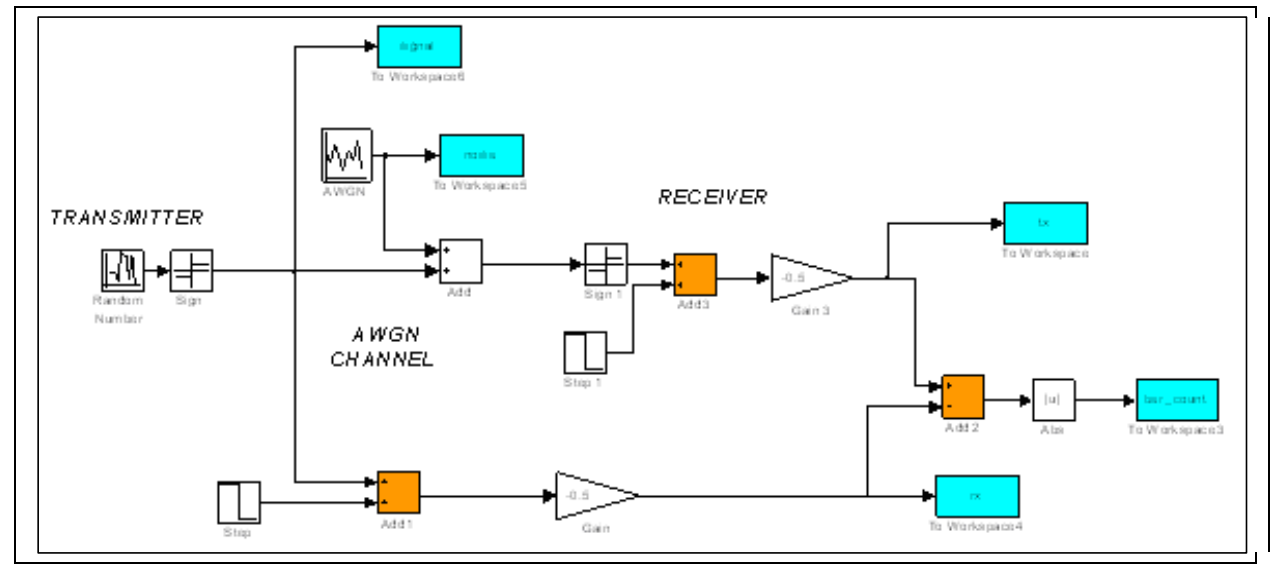

Figure 6. BPSK model

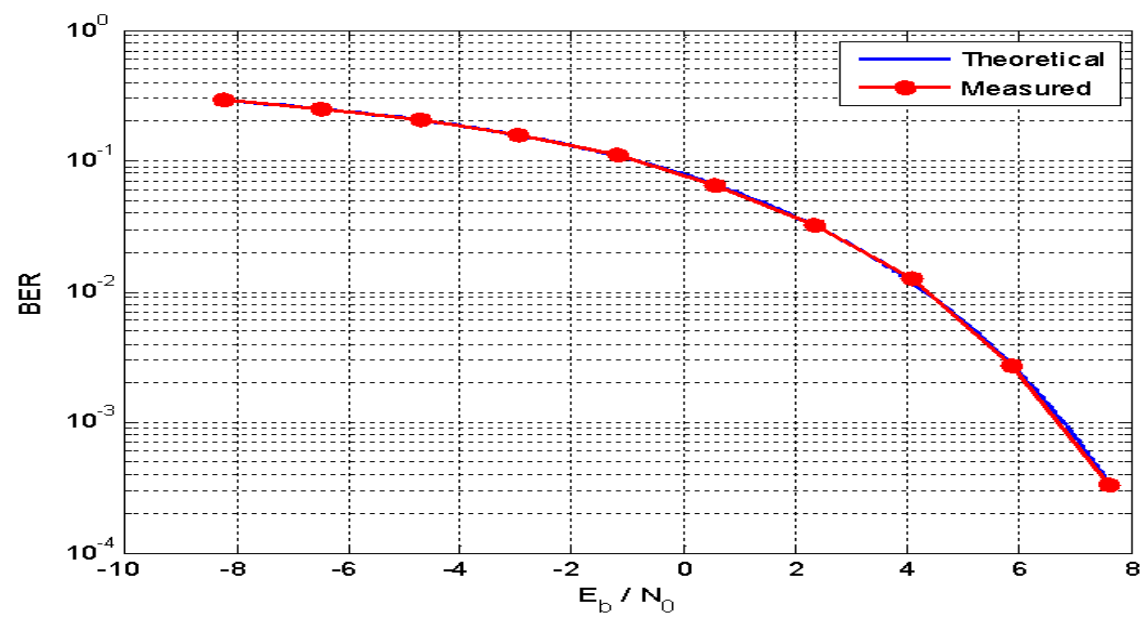

Figure 7. BER in AWGN channel

\subsection{Simulation Setup Model for BPSK using Fading Channel}

Here, the bit error rate at downlink with presence and absence of a Fading channel. In BPSK system with fading channel shown in Figure 8. 


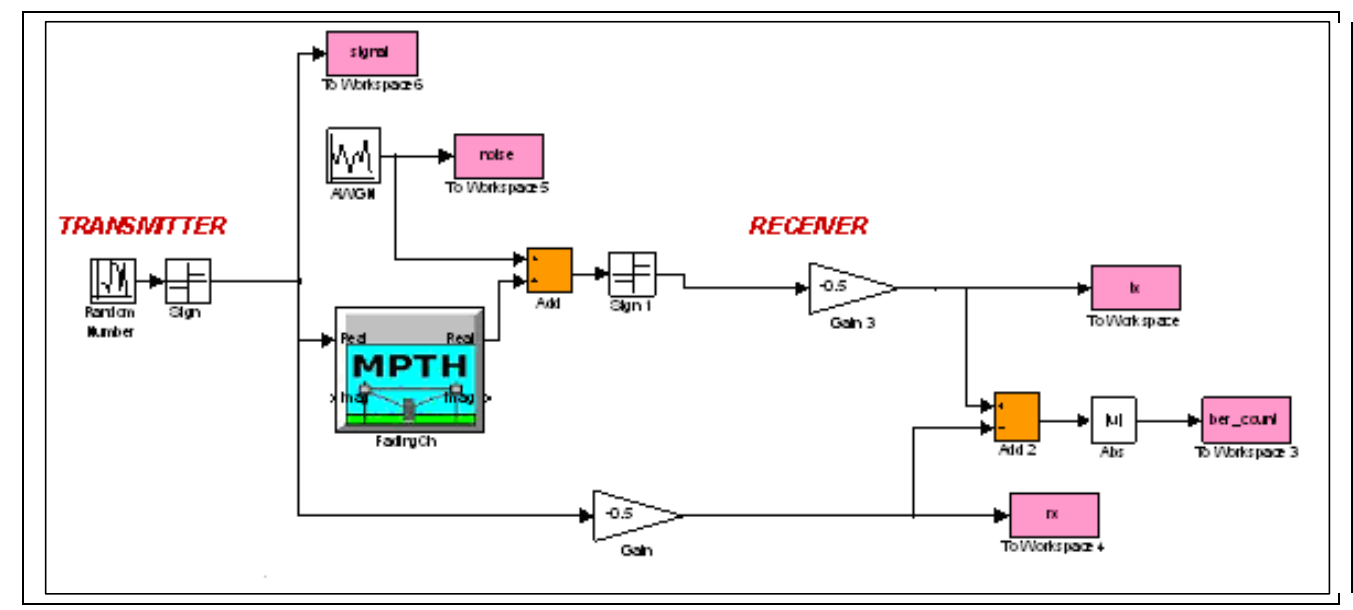

Figure 8. BPSK model with fading channel

The system performance with fading channel is $2 \mathrm{~dB}$ worst for BER of $10^{-2}$. The Figure 9 shows that BPSK model with fading channel can tolerate a $\mathbf{E}_{\mathbf{b}} / \mathbf{N}_{\mathbf{o}}$ of -10 to $8 \mathrm{~dB}$. The BER gets rapidly worse as the $\mathbf{E}_{\mathrm{b}} / \mathbf{N}_{\mathbf{o}}$ drops below $2 \mathrm{~dB}$. However, BPSK technique permits the Bit-Error-Rate to be more desirable in a noisy channel at the expense of transmission data capacity.

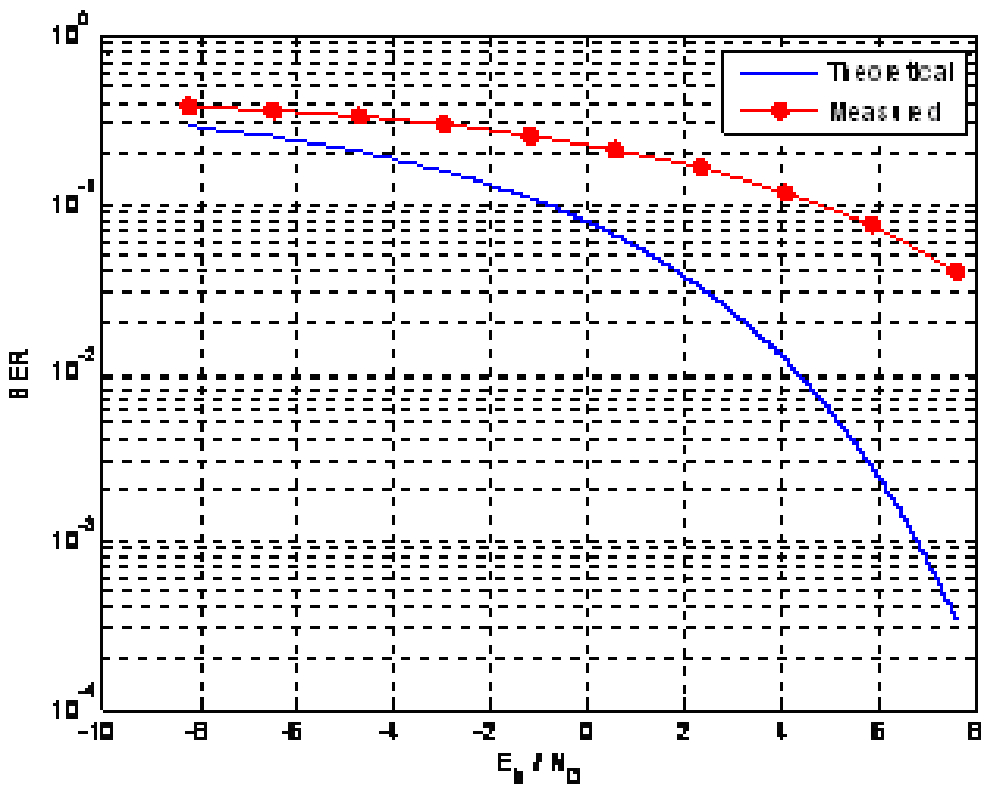

Figure 9. BER in fading channel

\subsection{Simulation setup model for BPSK using Moving channel}

The bit error rate at downlink with presence and absence of a Moving channel in BPSK system is shown in Figure 10 and Figure 11. The Figure 12 shows that BPSK model with moving channel can tolerate a $\mathbf{E}_{b} / \mathbf{N}_{\mathbf{o}}$ of $-9 \mathrm{~dB}$ to $1 \mathrm{~dB}$. BER gets rapidly worse as the $\mathrm{E}_{\mathrm{b}} / \mathrm{N}_{\mathrm{o}}$ drops below $6 \mathrm{~dB}$. 


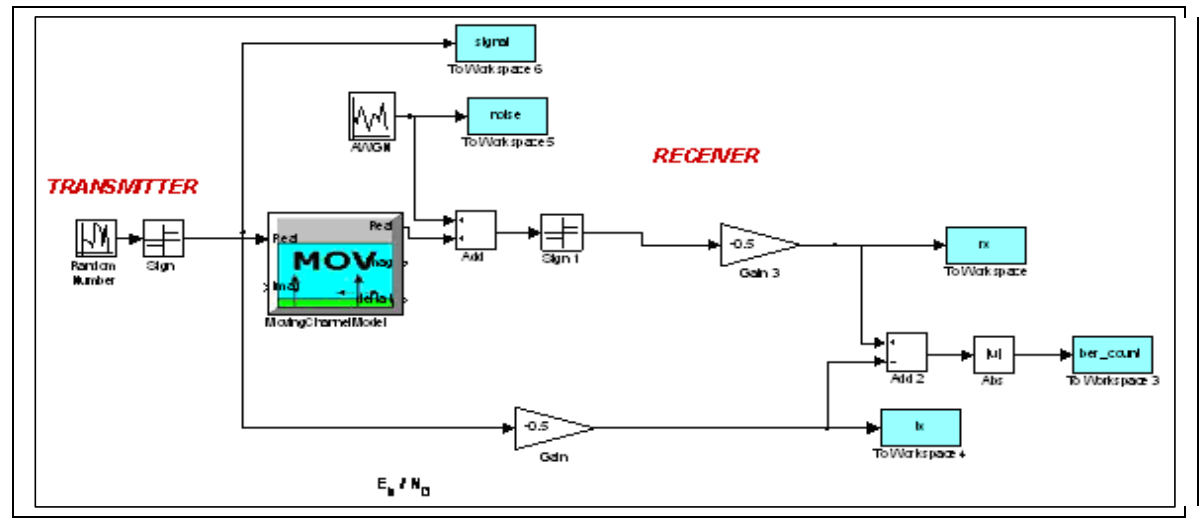

Figure 10. BPSK model with moving channel

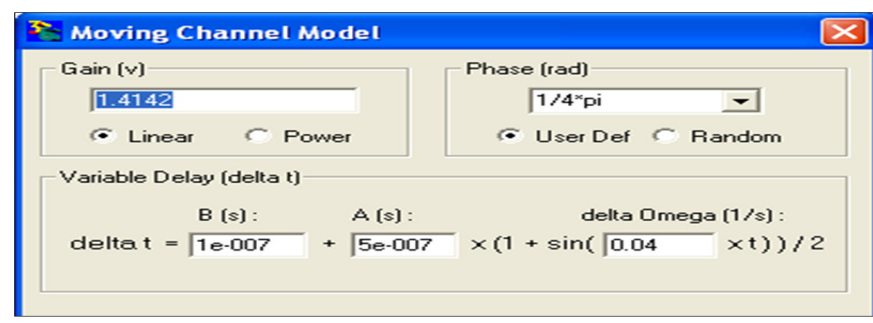

Figure 11. Moving channel model

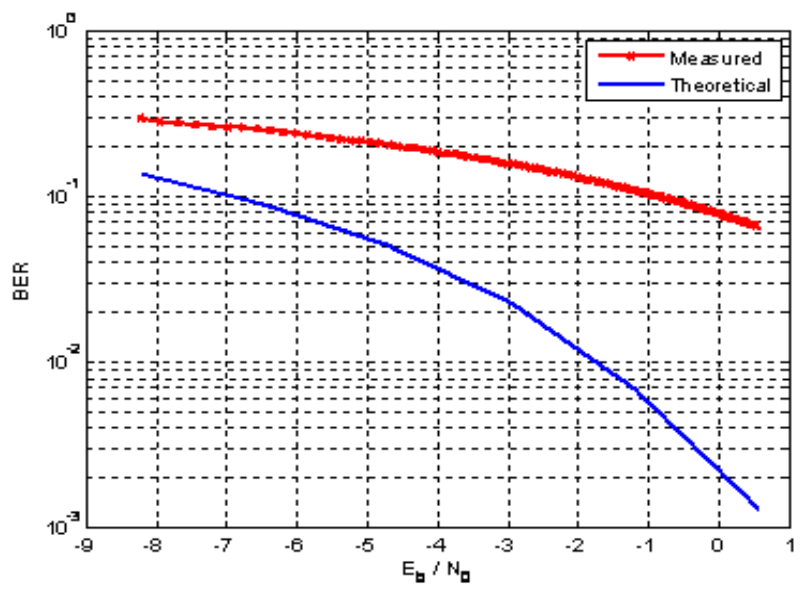

Figure 12. BER in the moving channel

\subsection{DPCH Generation using FDD block set}

The DPCH (Dedicated Physical Channel) is generated from two transport channels (DTCH at 64 kbps and DCCH at $2.5 \mathrm{kbps}$ ) and spread using the downlink spreading scheme as shown in Figure 13. This channel is dispread at the receiver, the slot format is demodulated, 2nd deinterleaving is applied and the two transport channels are demultiplexed. After channel decoding, the transmitted data is recovered. 


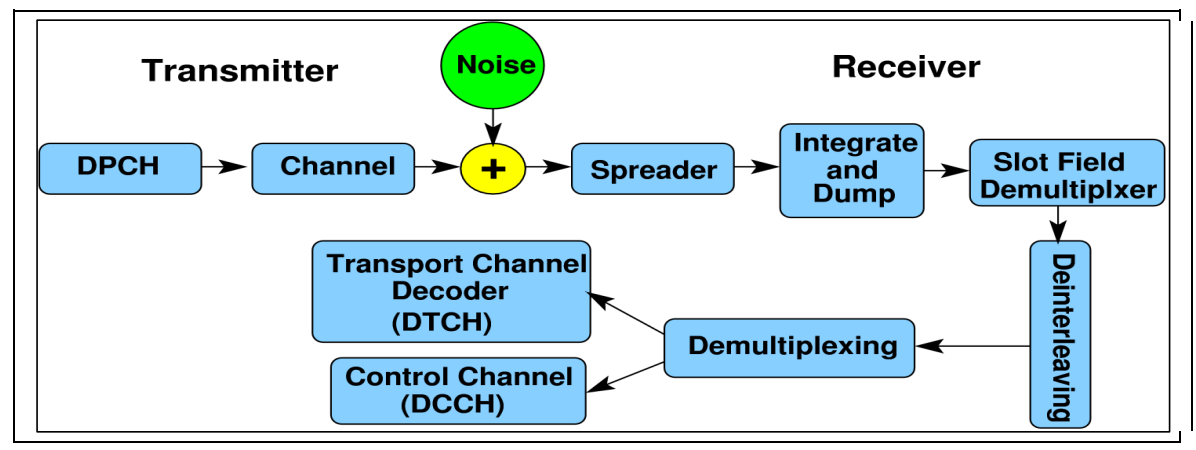

Figure 13. Block diagram of the dedicated physical channel

This model implements a 64 kbps DL reference measurement channel. This system transmits a dedicated transport channel (DTCH) at $64 \mathrm{kbps}$ and its associated control channel (DCCH) at 2.5 kbps (Figure 14). The aim of this module is to build this reference measurement channel and the specific functions to implement are:

- Transport channel generation

- Transport channel coding (cyclic code redundancy (CRC) coding; channel coding; rate matching; first interleaving transport channel multiplexing; second interleaving)

- Slot segmentation and mapping of transport channel into DPCH.

- Channelisation and scrambling.

\subsubsection{Transmitter}

Downlink Generator: This block produces all the downlink process including all channel coding, spreading/channelisation, rate matching and data framing, RRC filtering and final I/Q modulation onto a carrier.

\subsubsection{Receiver}

- Spreader/Despreader (Spreader): This stage is used to accomplish spreading and/or scrambling upon a baseband QPSK/BPSK signal for downlink process or uplink process. This stage can also be employed to achieve descrambling/ de-spreading.

- Integrate and Dump: This block can be employed at the despreader output in order to integrate and dump the despread chips over a bit/symbol period which is needed to recover the transmitted data.

- Slot Field demultiplexer: The Physical Channel Slot Demultiplexer demultiplexes the different fields of a physical channel slot and transmits the result into different outputs locations.

- Deinterleaving: $1^{\text {st }}$ stage or 2 nd stage permuting deinterleaver. Performs the inverse of the interleaver block

- Transport Channel Multiplexer: This block is used to create a multiplexer. This multiplexer can be employed basically in two cases in the FDD context. In the first case, the multiplexer can be employed to multiplex many logical channels such as DTCH, DCCH etc. into a transport channel (e.g. a dedicated transport channel DCH) before channel coding and interleaving. In this case, we may choose the transmission time interval (TTI) between the values $10 \mathrm{~ms}, 20 \mathrm{~ms}, 40 \mathrm{~ms}$ and $80 \mathrm{~ms}$. In the second case, 
The block can be used to multiplex up to 20 transport channels into a radio frame after channel coding. The TTI must not be confused with the radio frame length of $10 \mathrm{~ms}$. The TTI dictates the size of block over which channel coding takes place and the depth of the interleaving. The mapping from transport channels to physical channels splits the transport channel up into radio frames.

- Transport Channel Coder: This block is used to achieve the entire channel encoding/decoding that needed for a single transport channel in the uplink process and the downlink process.

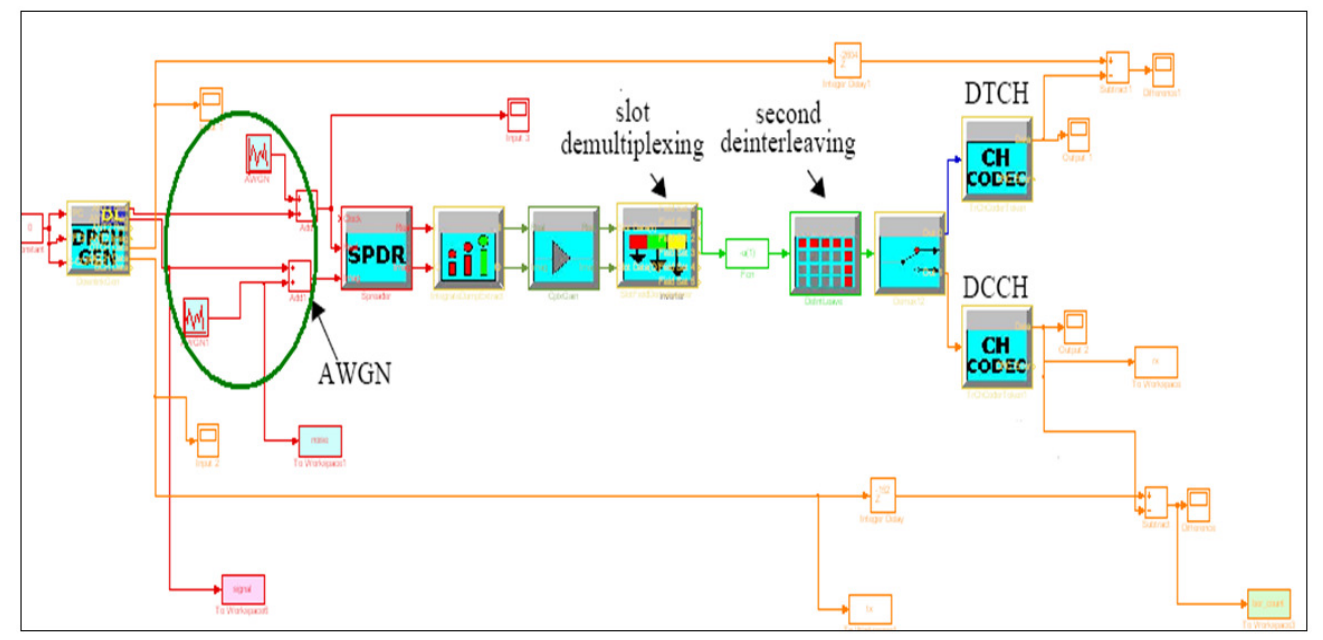

Figure 14. Dedicated physical channel

Table 1. Transport channel parameters

\begin{tabular}{|c|c|c|}
\hline Parameter & DTCH & DCCH \\
\hline Transport block size & $1280 \mathrm{bits}$ & $100 \mathrm{bits}$ \\
\hline TTI & $20 \mathrm{msec}$ & $40 \mathrm{msec}$ \\
\hline Error protection & Turbo coding & Conv. coding \\
\hline Coding rate & 0.33 & 0.33 \\
\hline Rate matching & $103.20 \%$ & $103.30 \%$ \\
\hline CRC size & 16 bits & 2 bits \\
\hline
\end{tabular}

Table 1 shows the simulation parameters for the setup model. By modifying the above parameters, then we can find the best combination to obtain higher performance of receiver in DPCH system.

\subsubsection{Channel Coding}

FDD systems have provision for both Convolutional Coding and Turbo Coding for error correction. For standard services that need BER equal to 10-3 convolutional coding is to be applied. The constraint length for the proposed convolutional coding schemes is 9. For services that need BER from $10^{-3}$ to $10^{-6}$, turbo coding is required. Reference provides a detailed description of the error correction coding schemes along with rate matching, interleaving and 
International Journal of Next-Generation Networks (IJNGN) Vol.4, No.3,September 2012

transport channel mapping. We will apply a rate $1 / 3$, constraint length 9 convolutional coding schemes to an uplink $9.6 \mathrm{kbps}$ voice service and show the BER improvement as a result of coding gain.

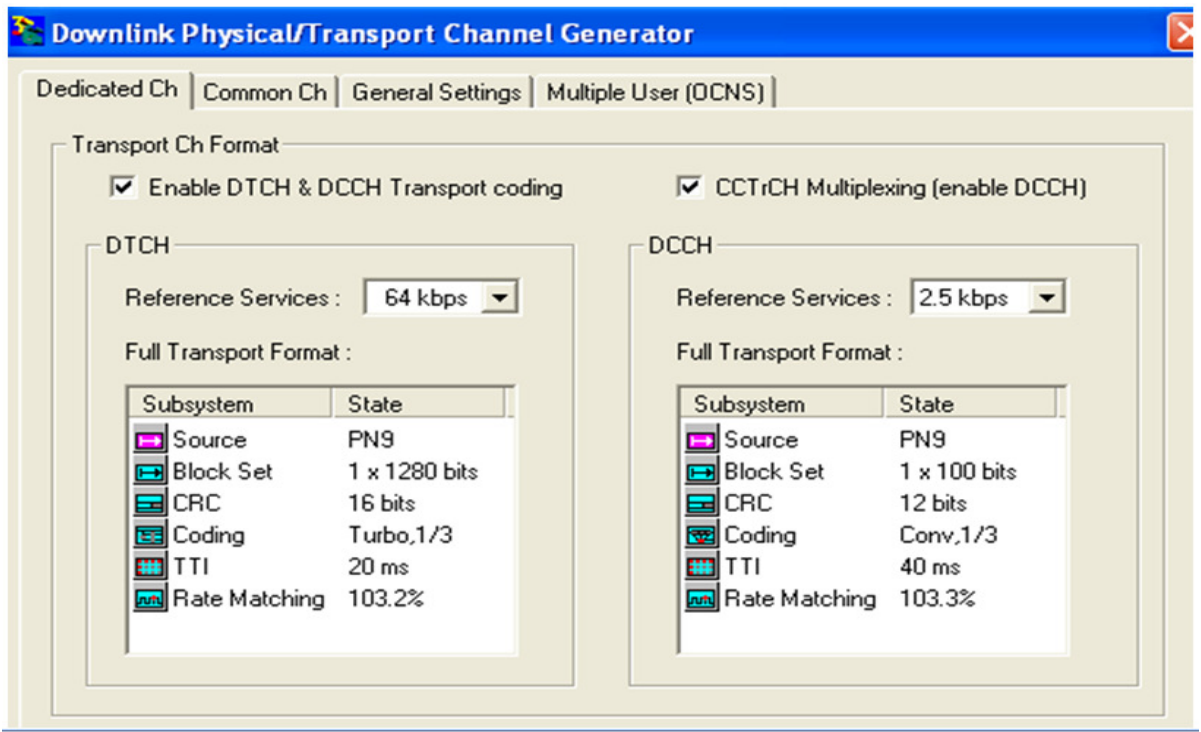

Figure 15. Transport channel format

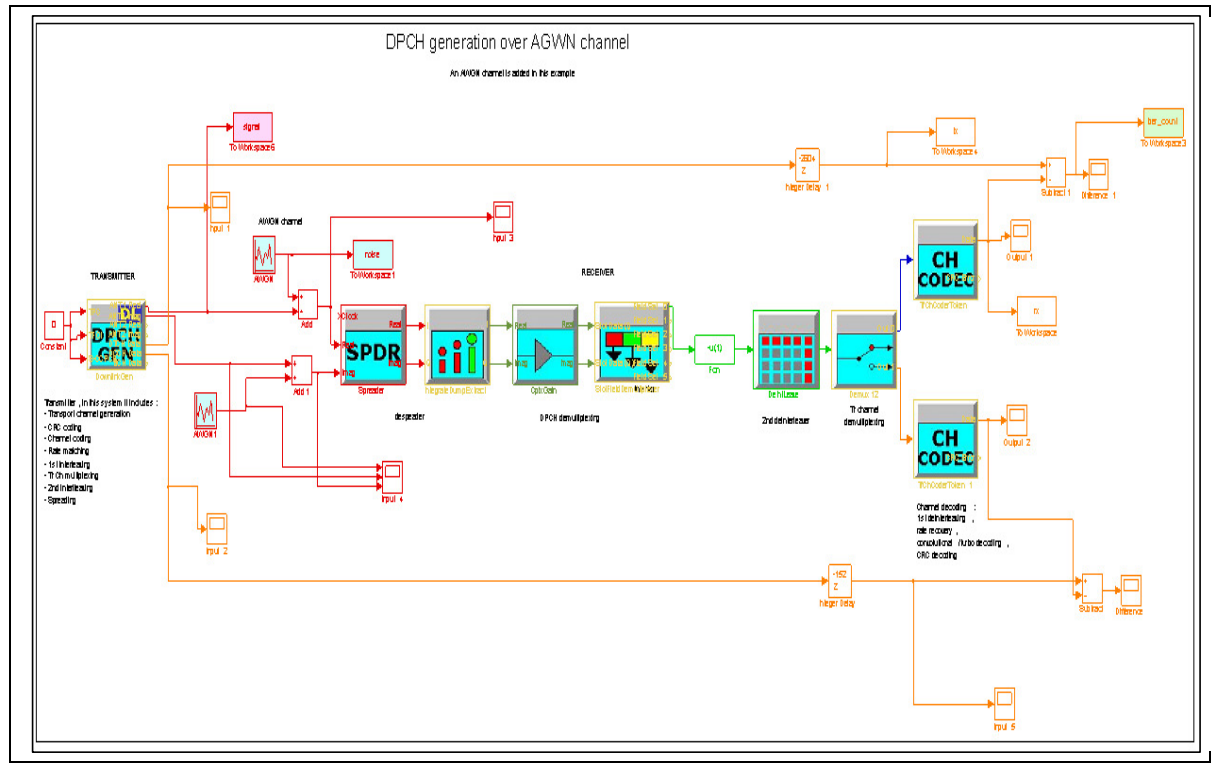

Figure 16. Calculate BER for DTCH 


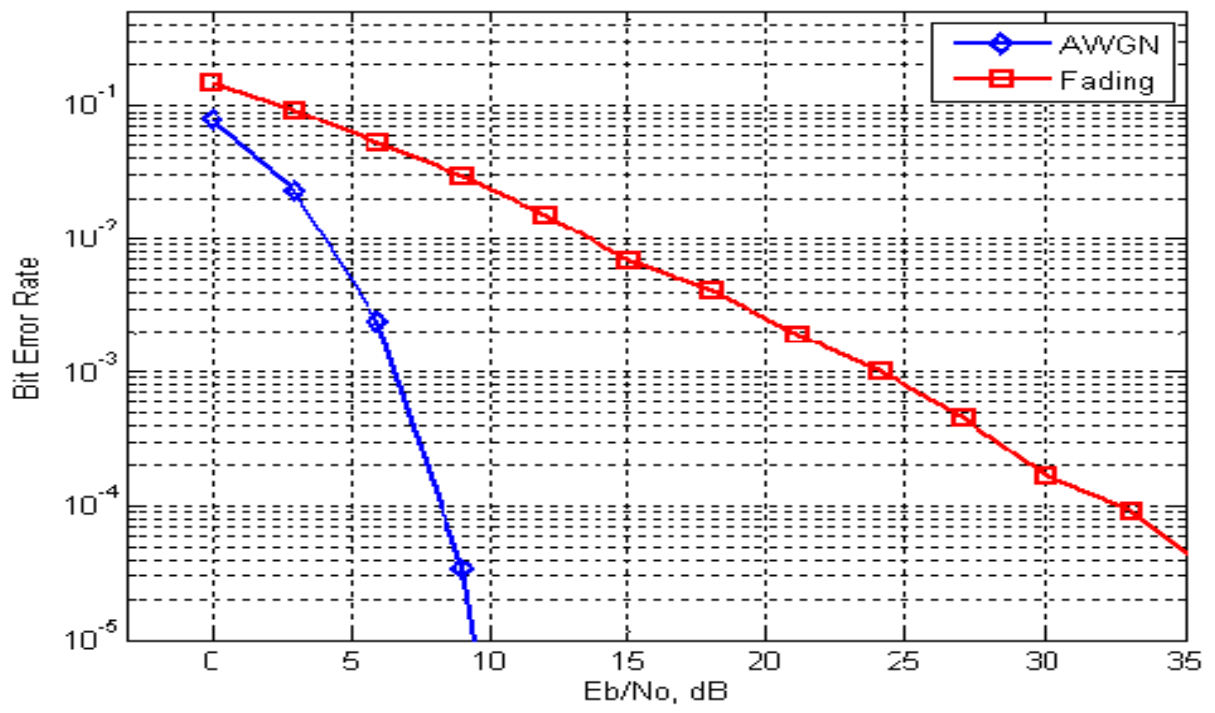

Figure 17. BER for DTCH

The model shown in the Figure 18 is employed to calculate BER for dedicated control channel (DCCH).

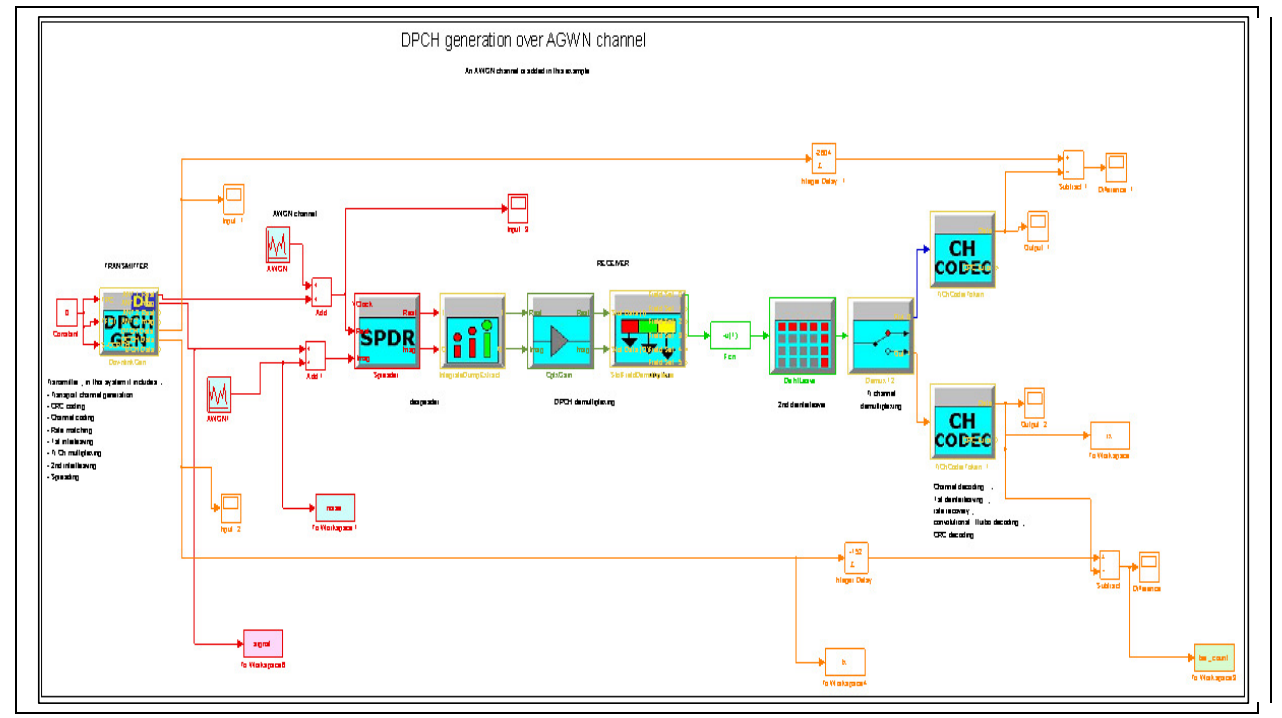

Figure 18. Calculate BER for DCCH

The performance of the receiver in case of UTRA $3 \mathrm{G}$ fading channel is $5 \mathrm{~dB}$ worst as compared to AWGN channel as shown in the figures 17 and 19. 


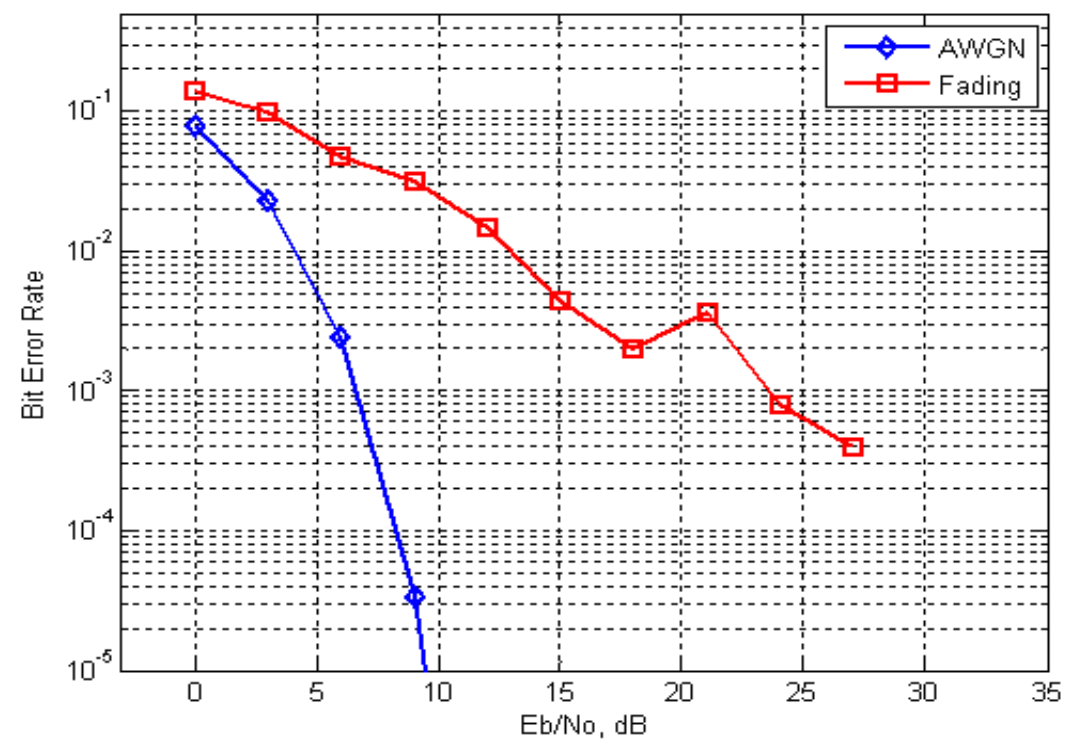

Figure 19. BER for DCCH

\subsection{BER Measurement using the BER Token}

In this system in the Figure 20, a constantly decreasing level of additive noise is used to simulate the situation where a receiver performance is improving over time. This is in order to highlight the difference between Block BER and Total BER. Note that high noise levels at the start of the simulation run cause the Total BER output to show a high BER that doesn't disappear completely by the end of the run, even although the noise by that time is minimal. By taking 10 bit trials, the Block BER shows the real situation better. By the end of the run, the Block BER shows zero errors as shown in Figure 21.

\subsubsection{BER Token}

This stage reflects the ability to evaluate the Bit Error Rate (BER) performance of physical layer simulations. The two inputs positions have to be linked to two data streams with the same rate. It would be equal under complete operation e.g. the input to a spreader and the output from a despreader. The inputs are quantised using the "Input Threshold" parameter. Input values above or equal to the threshold are defined as "high", and conversely values lower than the threshold are defined as "low". The occurrence of a bit error is found by the logical XOR of the two input values after thresholding. The resulting sequence of bit errors from the logical XOR is then manipulated for the three outputs. The "Total Errors" output is simply a running total of the number of bit errors since the beginning of the system run. The "Total BER" output can be evaluated by dividing the total number of bit errors over the number of bits tested, and is consequently a measure of the probability of a bit error averaged across the system run. The "Block BER" is the number of bit errors in the current block divided by the length Parameter Range Default value Definition. When calculate total BER in AWGN take the average of total BER and change Eb/No from 10dB to $50 \mathrm{~dB}$ as shown in Figure 22. 


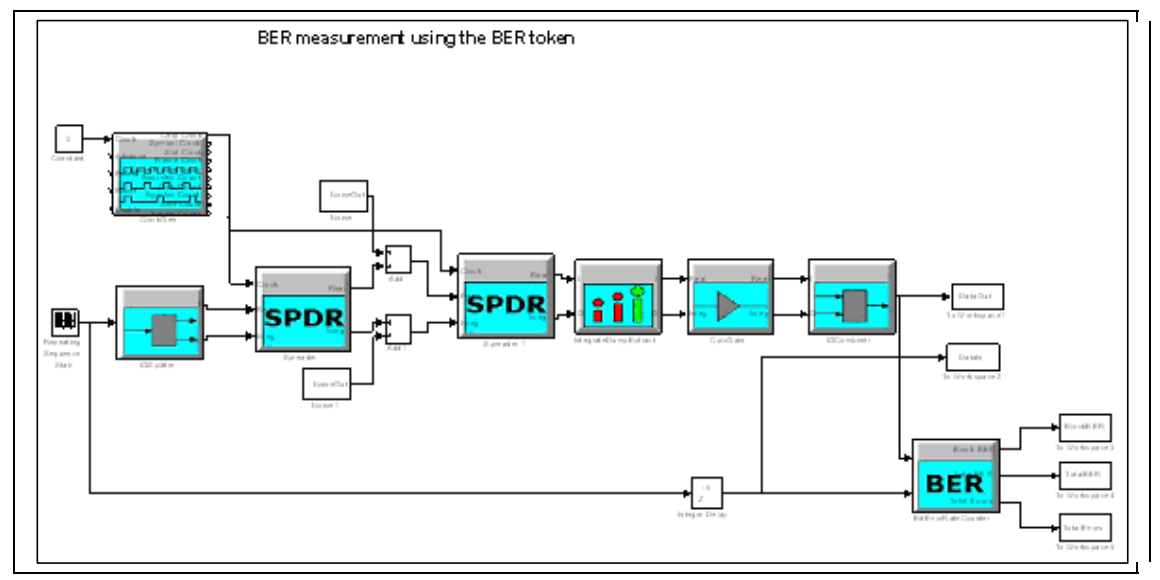

Figure 20. BER measurement using the BER token

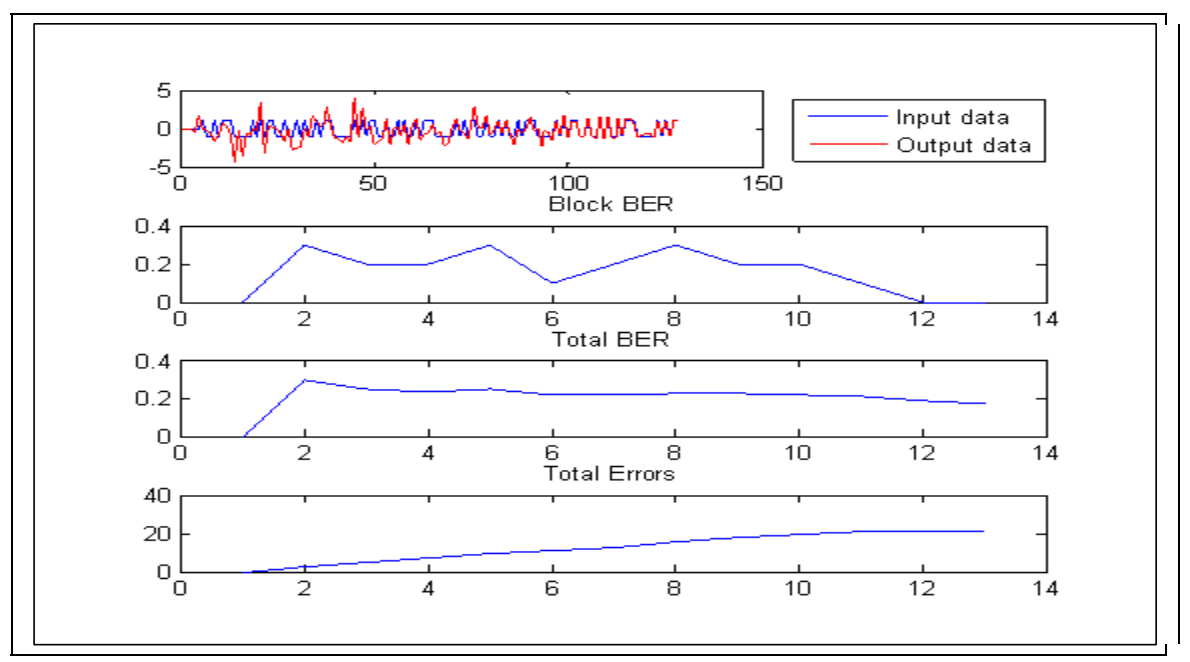

Figure 21. Output of BER token

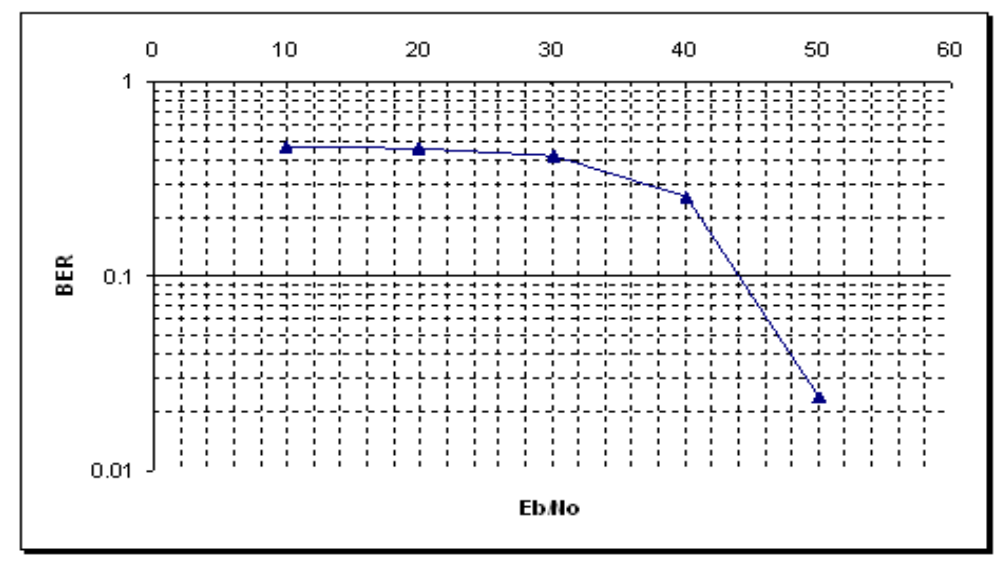

Figure 22. BER measurement using the BER token 


\section{Conclusions}

At the end of this work, we conclude that the benefits of using the FDD library for rapid prototyping, system modelling and practical training have been presented. The performance of the receiver in case of UTRA $3 \mathrm{G}$ fading channel is $5 \mathrm{~dB}$ worst as compared to AWGN channel. The performance gets worst with increasing number of multipaths and phase difference (between different multipaths). Also the UTRA FDD library can be employed with many blocks from other Simulink libraries, such as the DSP and Communications block sets, so that more complex, proprietary models can be realised.

\section{REFERENCES}

[1] I. E. Consortium, Cellular communications, Web Proforum Tutorials - http://www.iec.org, 2004.

[2] G. Williams, Cellular communication networks, Lehigh Unversity, 1995.

[3] GeekInterview, What is $1 \mathrm{~g}$ - rst generation, http://www.geekinterview.com ,2005.

[4] C. N. Perspectives, The amps family of wireless standards, http://www.cnp-wireless.com, 2007.

[5] F. Khan, LTE for 4G Mobile Broadband- Air Interface Technologies and Performance . rst ed., 2009.

[6] G. Tsoulos, MIMO System Technology for Wireless Communications . 2006.

[7] 4GWirelessEvolution, Nokia siemens networks demonstrates lte interoperability on qualcomm platform, http://4g-wirelessevolution.tmcnet.com

[8] 3GPP, Third generation partnership project, http://www.3gpp.org , 2008.

[9] 3GPP, 3gpp ts 25.104 v3.5.0(2000-12), 3rd Generation Partnership Project, 2000.

\section{Author: IBRAHIM AREF}

\section{ACADEMIC QUALIFICATIONS}

- Doctor of Philosophy $(\mathrm{PhD})$ in System Design and Modeling, Electronic and Electrical Engineering, University of Glasgow, February 2011.

- Master of Science (MSc) in Computer Systems Engineering, University Putra Malaysia (UPM), Faculty of Engineering, Computer and Communication Systems Engineering Department, March 2004.

- B.Sc. in Computer Engineering, Al-Fateh University, Faculty of Engineering Computer Engineering Department, July 1993.

\section{CURRENT POSITION}

Lecturer in Computer Engineering Department, University of Tripoli-Libya. 